\title{
Designing Personally Relevant Avatars for Digital Health Interventions: The Biocultural Perspective of Presence
}

\author{
Polina Durneva \\ Department of Information Systems and Business \\ Analytics \\ Florida International University \\ pdurn001@fiu.edu
}

\author{
Cynthia LeRouge \\ Department of Information Systems and Business \\ Analytics \\ Florida International University \\ clerouge@fiu.edu
}

\begin{abstract}
Digital health interventions (DHIs) show great promise in empowering patients to take positive action toward their self-care by helping them with chronic disease self-management efforts. However, problematic user engagement with DHIs is a key issue preventing the full realization of DHI benefits. DHI design issues, such as lack of personal relevance, can negatively impact user engagement and consequently prevent patients from entering the empowerment process. The literature recognizes that avatars can be used to assimilate a self-concept during humancomputer interaction and enhance personal relevance through self-presence. Yet, little is known about designing avatars to achieve self-presence in the context of digital health. This paper reports the results of a design science research study that explores key design elements that can facilitate a personal connection between users and technology by inducing self-presence through avatars. This study has implications for the theory-driven design of DHI to engage users with chronic conditions.
\end{abstract}

\section{Introduction}

Chronic disease is the leading cause of death and disability as well as the leading driver of annual healthcare costs ( $\$ 3.5$ trillion in annual healthcare costs (2019)) in the US [1]. Digital health interventions (DHIs), digital health that delivers health interventions via smartphones, websites, and text messaging [2], hold great potential for patient self-management of chronic health conditions [3]. Behavior change (e.g., eating healthy) is critical for chronic disease management and prevention [4]. DHIs for self-management of chronic conditions are increasingly gaining attention to drive changes in user's attitudes, behaviors, and compliance behaviors that could lead to improved health outcomes $[5-12,79]$. In this study, we focus on health behavior change, one of the major goals of health behavior support systems [4, 79].

Considering user engagement in the healthcare context may be particularly important for achieving the promise of digital health interventions [13]. In Human-Computer Interaction (HCI), user engagement is conceptualized as the subjective experience of flow, a mental state characterized by enjoyment and focused attention [14]. User engagement can provide a powerful explanation for intention to use and continued use of technology [15-17].

User engagement has been associated with the health intervention effectiveness of digital health technologies, specifically health behavior change [1821]. However, studies report that problematic user engagement is one of the major challenges in realizing the full potential of digital health interventions [21-27].

Problematic engagement with DHIs can be attributed to design issues. Studies recognize that personal relevance is one of the desired DHI characteristics (as reported by DHI users) that can improve user experience and enhance engagement [2830]. Personal relevance can be described as a personally meaningful connection between user and technology [31]. In the field of persuasive technology, there is the general thought that the more users identify with technology (i.e., the more users find technology personally relevant for them), the more likely they will be persuaded to change their behaviors in a way that the technology suggests [24 32]. Even though personal relevance is essential for both engagement and the ultimate target of behavior change, there is currently more emphasis on designing technically sophisticated DHIs than making these technologies personally relevant to users [33]. As a result, most existing DHIs fail to feel relatable to their users [29 33], which may 
cause problematic user engagement with DHIs. Creating personally relevant DHIs can improve user engagement with DHIs thereby leading to health behavior change and improved health outcomes.

Therefore, we need to understand how to support the personal relevance of technology through various design features. In this study, we leverage the concept of self-presence, an illusion that one's virtual representation is indeed oneself [34], to address this question. Research indicates that user's experience of self-presence is associated with enhanced levels of perceived personal relevance of technology [35].

Self-presence can be realized through avatars, users' virtual representations that facilitate interaction with the virtual environment, entities, and other users [36-37]. Self-presence functions by mapping users onto their avatars [38] and converging the virtual with the physical through the interplay of the user's offline and online identities [39]. Designing avatars that allow users to achieve self-presence can increase the personal relevance of technology to its users and cause an effective behavior change [40].

Design Science Research (DSR) may be the best methodological perspective to expand the current limits in knowledge and understanding regarding the design of digital health avatars. This methodological perspective focuses on creating innovative artifacts (e.g., methods, models, constructs, instantiations) and knowledge about artifacts at different levels of abstraction [41-42]. In DSR, design principles are one of the main forms of design knowledge [41-45] that capture the knowledge "... about creating instances of artifacts that belong to the same class" [46]. Design principles are prescriptive statements that explain how to do something to achieve a specific goal [47]. Design principles put a lot of emphasis on the user's social context [48]. Understanding user's social context can be crucial for creating tools to support health behavior change [49]. Applying design principles to DHIs can be powerful in terms of understanding potential users (e.g., their life experiences related to illness) and helping users achieve their health behavior goals. At present, design principles that provide direction in the DHI and general IT design space to enhance selfpresence through avatars do not exist. Existing studies on avatar design either do not focus on design principles at all [50] or focus on design principles that do not capture the concept of self-presence and the context of health [51].

The purpose of this study is to develop design principles to prescribe how to design avatars that help users achieve self-presence in their use of DHI to selfmanage chronic conditions. To inform the design principles for avatars in DHIs, we draw on the biocultural theory of presence [52]. The biocultural theory of presence is appropriate for understanding self-presence in the context of chronic care because it explicates multiple layers of self-presence (body, emotional, identity) that align with the social context of chronically ill users.

Researchers and developers can incorporate evidence-based design principles to support avatarinduced self-presence in the avatar design. In addition, decision-makers can make better selections among alternative DHIs. Avatars developed and deployed that align with the self-presence design principles can enhance the personal relevance of DHIs for its users. Improvements in the DHI design have implications for engagement with technology and, therefore, positive health behavior changes, which can eventually lead to improved health outcomes of the chronically ill.

\section{Background}

\subsection{Design Principles: Key Aspect of Design Science Research}

DSR is a problem-solving approach that focuses on creating a novel artifact and knowledge about the artifact [41] at different levels of abstraction, ranging from concrete instantiations to design principles and design theories [42]. Design theory is prescriptive and emphasizes theoretical underpinning, which says how a design process should be carried out in effective and feasible ways [74]. The purpose of design theory is to support the achievement of specific goals by attending to meta-requirements (the class of goals to which the theory applies) and meta-designs (a class of artifacts that meet the meta-requirements) of the artifact.

The scope of this study is to generate a set of design principles, a well-known and accepted form to communicate nascent design knowledge in design theory [43], by attending to the social context of key stakeholders and the overall purpose of the artifact. Design principles are statements that guide or constrain actions, prescriptive in nature, and constitute the basis for action [47].

DHIs that are based on design principles can be a powerful tool to drive health behavior change. Design principles put a lot of focus on the user's social context [48]. In health-related research, social context refers to a patient's life experiences, social relationships, and societal influences [75]. Understanding user's social context can be crucial for creating tools to support health behavior change [49]. For example, a user might be lacking social support to achieve their healthy eating goals. Design principles could reveal this aspect of user's lives and provide guidance on how to address this issue through technology design. 
There currently exist no design principles that guide the design of avatars to enhance self-presence in the context of chronic care. First, existing studies on design principles for avatars (e.g., [51]) do not focus on the context of health or more specifically chronic care. For instance, Blake and Moseley (2010) discuss general design principles for avatars in educational technology [51]. Design principles for educational technology might not be completely pertinent to the goals and context of DHI users. The goal of educational technology lies in facilitating learning (e.g., avatars providing users with links to relevant information sources) and supporting the cognitive efforts of users. On the other hand, DHIs go beyond facilitating learning as they aim to promote some level of understanding of healthy behavior and also induce health behavior change (e.g., showing users how to perform physical activity). In addition, the social context of DHI users revolves around their health and well-being that might not be necessarily relevant to users of educational technologies. For DHIs, it is crucial to capture the nuanced experiences of using technology for health to enhance the personal relevance of technology for this population.

Second, in the context of health, avatar design has been explored [69] but not in relation to self-presence. There is still a lack of research that prescribes how to induce self-presence in DHIs. Some DHI studies tend to focus on specific avatar characteristics (e.g., similarity, avatar body size) and the effectiveness of DHIs [50]. Other studies tend to look at the relationship between self-presence and avatar-induced behavioral conformity (i.e., Proteus effect) [39] or the avatar-induced behavioral conformity and behavior outcome (e.g., physical activity and eating behaviors) [40] omitting explication of how to holistically design avatars in DHIs to induce user's sense of self-presence.

To address gaps in current research, we need to create design principles that account for the concept of self-presence and are appropriate for avatars in healthcare. To achieve this goal, we leverage the biocultural theory of presence [52]. This theory views the concept of presence as having three layers: proto, core, and extended. These layers can reflect avatar design with respect to avatar body, avatar emotions, and avatar identity. Moreover, these layers are appropriate for the context of healthcare as they align with the experiences of chronically ill users who experience their illness in terms of their physical functioning, psychological functioning, and illness identity [54].

\subsection{Biocultural Theory of Presence}

The biocultural theory draws on Damasio's theory of consciousness [53] by conceptualizing natural (inperson) and technology-mediated presence in terms of proto, core, and extended presence [52]. The tight integration of three layers enhances the overall sense of presence.

These three layers of presence (proto, core, and extended) align with the following experiences of the chronically ill: disruptions in physical functioning, disruptions in psychological functioning, and formation of illness identity [54].

Proto presence refers to the sense of physical being based on the extent to which individuals can correctly couple their perceptions and movements and, therefore, differentiate themselves from the external world [52]. In the context of avatars, proto (body-level) self-presence has been defined as "the extent to which a mediated self-representation is integrated into body schema" (p.325) [55]. When proto self-presence is achieved, avatars become a physical extension of the body, and awareness of the gap between a user and technology dissipates.

Proto self-presence aligns with the user's physical functioning, which refers to the individual ability to perform various daily living activities [56 57]. Individuals with chronic conditions may experience changes in their appearance, and their experiences of various sensations in the body may interfere with daily activities, thereby affecting their physical functioning [58]. To derive design principles at the proto level, we propose the following research question:

\section{Research Question 1: What are the design principles for avatars at the proto level of self-presence that reflect user's physical functioning?}

Furthermore, core presence refers to individuals' emotional state that arises from the encounters between proto self and objects in the external environment [53]. In the context of avatars, core (emotion-level) selfpresence has been defined as "the extent to which mediated interactions between self-presentation and mediated objects cause emotional responses" (p. 326) [55]. Emotional responses arise during the experience of technology use and may linger after the experience for some time.

Core self-presence can be aligned with user's psychological functioning. For chronically ill users, physical limitations and changes can lead to individual distress [59], resulting in a person's disruption of psychological functioning, i.e., emotional well-being [60]. Disruption in the psychological functioning of the chronically ill can be associated with "fear that one is 
losing control over one's body and/or that one can no longer do what one could before" [58] and diminishing trust in one's own body. Therefore, to understand how to design avatar at the core level, we propose the following research question:

\section{Research Question 2. What are the design principles for avatars at the core level of self-presence that reflect user's psychological functioning?}

Finally, extended presence, which Damasio calls as autobiographical self [53], refers to the individuals' perception of their identity based on personal traits and past experiences [52]. In the context of avatars, extended (identity-level) self-presence has been defined as "the extent to which some aspect of a selfrepresentation is related to some aspect of personal identity" (p.326) [55]. Illness identity, which defines the degree to which an illness becomes integrated into one's sense of self [54 58 61], forms as a result of changes in physical and psychological functioning and aligns with extended self-presence. To understand how to design an avatar at the extended level, we propose the following research question:

\section{Research Question 3. What are the design principles for avatars at the extended level of self-presence that reflect user's illness identity?}

Overall, in this study, we aim to identify design principles for avatars that allow users to achieve selfpresence in DHIs for self-management of chronic conditions. By leveraging the biocultural theory of presence, we create design principles that align with the life experiences of chronically ill users at body, emotion, and identity levels. To achieve this goal, we draw relevant data from a user-centered design science project that sought to design DHIs for managing obesity and overweight.

\section{Methods}

\subsection{Context of the Study}

The context of this study is the development of digital health to support overweight and obese adolescents. Adolescent overweight and obesity are major public health issues affecting more than 12.7 million children and adolescents and the overall healthcare system ( $\$ 190$ billion in annual healthcare costs in the US) [62-64]. It is particularly relevant to study adolescent digital health as adolescents tend to be early adopters [65] and the highest users of technology [66]. DHIs have great potential to improve the health behaviors of obese and overweight early in life. Healthy behaviors developed early in life can set the foundation for lifelong health [67 68]. Moreover, research shows that adolescents are generally interested in having virtual characters to assist adolescents with self-management efforts in the context of digital weight management programs [6971].

The current study draws relevant data from a larger user-centered design science research project aimed at designing a DHI targeting obese and overweight adolescents. For this study, we focus on aspects of the methods and data mainly related to the inclusion of avatars in the design of DHIs to support overweight and obese adolescents in self-management. Further details about focus groups, semi-structured interviews, usability testing, and coding procedures can be found in prior studies [69-72].

The data for this study were collected in two phases, including focus groups and usability testing with adolescents participating in structured healthy behavior programs. Phase 1 techniques included 10 one-hour user-driven focus groups with adolescents participating in accredited weight self-management programs. Phase 2 focused on usability testing of screen prototypes and exploring the context of use. 70 adolescents attending the weight management camp sessions (Camp Jump Start) were recruited to participate in usability sessions that lasted approximately 1 hour. Qualitative data from Phase 1 and Phase 2 were analyzed using Dedoose $($, a qualitative data analysis software. Usability testing results from Phase 2 were used to inform our research questions.

\subsection{Grounded Theory Methodology Techniques}

There exist four main grounded theory methodology (GTM) approaches: the classic approach, the evolved approach, the mixed-method approach, and the use of GTM techniques for data analysis [76]. In this study, we use GTM techniques for data analysis. This approach employs GTM coding techniques for data analysis without strict adherence to GTM principles. In line with this approach, we use the biocultural theory of presence as a priori theory to guide open and selective coding techniques towards deriving a set of theory-driven principles.

First, we revisited transcripts and notes related to avatars from the usability testing sessions (Phase 2). We first applied open coding to break data down analytically and discern which sections of transcripts and notes were relevant to the current study.

Specifically, we looked for meanings and concepts that were direct or tangent to the understanding of the 
biocultural theory's aspects and the social context of obese and overweight.

Second, we analyzed relevant coding structures by applying selective coding procedures to develop a set of concepts to provide a theoretical explanation of the phenomenon of interest [77]. During selective coding, all categories of data are unified under core categories, which represent the central phenomenon of the study. In accordance with selective coding, we mapped data related to features and the context of avatars' use to higher-level concepts from biocultural theory.

During the coding process, the team met regularly to iteratively discuss initial coding relevant to the current study and reach a consensus regarding identified themes identified in selective coding [78]. Reconciling issues were minor and primarily consisted of agreeing on labels for identified themes. Data analysis was complete after the team had reached a consensus that the resulting themes related to each selfpresence layer were accurate and stable.

\section{Results}

Overall, adolescents' responses reveal various avatar features that align with three self-presence layers: proto, core, and extended. Proto self-presence design principles align with user's physical functioning and show avatar features at the body level. Core selfpresence design principles align with user's psychological functioning and show avatar features at the emotion level. Finally, extended self-presence design principles align with user's illness identity by showing avatar features at the identity level.

\subsection{Proto self-presence}

Participants expressed that avatars could act as their proxy in the virtual environment. The adolescents also identified specific body schema design elements requiring consideration to facilitate proto self-presence and align with their physical functioning: alignment between appearance and behavior, voice, physical body, and body movement.

Universally, the adolescents sought alignment between the avatar's appearance and behavior. For example, one participant felt that the animal-looking avatars should be designed to say things and perform actions that are "animal appropriate," therefore, a dog would not roar like a lion or run like a horse.

The adolescents discussed the physical body representation of the avatar in relation to matching their own anthropometric measurements such as weight and height. For example, one participant said, "What your avatar weight is, and then you work from that and you can change the weight, make it lower, like you eat healthier, your avatar gets thinner too while you get thinner."

Most adolescents felt that an avatar with a voice would prompt them towards behaviors that would support their health goals (e.g., interactions with other avatars or receiving health tips). The manifestation of a voice could be expressed by a visual speech bubble, audio sound, or both.

Finally, participants indicated that body movement would be relevant to their avatars. Participants stated that they would be interested in seeing the avatars move their arms and legs in some way. Participants suggested various basic actions that can be performed with avatar's arms and legs: walking, running, jumping, shaking head yes or no, clapping, and pointing. They also suggested more complex movement patterns aimed at performing tasks such as cooking, dancing, and eating. One participant said, "And that person is doing the cooking and the clean-up so it kind of shows you a little bit how to do this in a certain way that you're supposed to do it."

In alignment with adolescents' responses, we formulated the following design principles:

To allow chronically ill users to achieve proto self-presence, avatars in DHI for self-management of chronic conditions should...

DP1: ......exhibit alignment between appearance and behavior.

DP2: ...match users' anthropometric measurements such as height and weight.

DP3: ...interact with users (through a voice feature).

DP4: ...perform a range of body movements.

\subsection{Core self- presence}

Participants discussed several avatar features that align with core self-presence and psychological functioning of users. Discussed features were related to the motivational content, facial expressions, and emotional responses.

Participants discussed several ways in which avatar could provide users with motivational content that could affect their motivational state in relation to engagement with technology and health behaviors. Regarding content, some participants were interested in avatar's instant encouragement in the form of motivating messages (e.g., "Good job!") as a response to users' actions. Different elements of the reward system (e.g., badges, labels, new accessories, and clothing) were mentioned as forms of motivational content to be engaged with technology. 
Also, participants discussed avatar's ability to express emotions in response to users' actions. Some participants mentioned specific facial expressions (e.g., looking angry, frustrated, curious, or confused). For instance, one participant said, "If you're just eating pizza and ice cream it [avatar] would be all [...] like sad, frowny face."

General emotional responses in reference to users' food choices were also indicated. For example, one participant said, "Happy, never really angry, but it [avatar] can become like mad a little bit, you know? If you're not doing it for more than a week, it could become angry?"

In alignment with adolescents' responses, we formulated the following design principles:

To allow chronically ill users to achieve core selfpresence, avatars in DHI for self-management of chronic conditions should...

DP5: ...provide motivational content.

DP6: ... have facial expressions in response to users' actions.

DP7: ... provide emotional responses to users' actions.

\subsection{Extended self-presence}

Participants identified various avatar features that could contribute to the ongoing construction of an individual self (e.g., their identity) and align with extended self-presence. These features appear to support adolescents' presence in the virtual environment as envisioned or historical selves and could, therefore, facilitate the extended self-presence. These features included appearance, character, future self, environment, avatar-friend, and avatar-coach.

Participants generally stated that they want to express themselves in the virtual environment by changing their avatars' appearance. Preferences in terms of the degree of likeness to self-varied. Some adolescents indicated that they want to take a picture of themselves and create their avatars based on their actual appearance. Other participants of the study emphasized that they wanted an avatar to resemble themselves at least partially.

Moreover, participants discussed their interest in choosing different characters (e.g., chefs, teachers, trainers, etc.) for their avatars. For instance, one participant said, "I'd say make like a character or something like a main character like a chef or something."

Participants also discussed various features pertaining to their vision of the future self in relation to their appearance and health. Some participants wanted their avatars to decrease in size as new weights or foods are entered in the application to align with what the adolescents are experiencing or want to experience in real life as they change their life habits. For instance, one participant said, "Well, I think there should be an avatar of what you look like now and what you want to look like, so, I think that would be really cool."

Furthermore, participants commented about the environment. Specifically, participants wanted to align their self-representations with their virtual surroundings. Participants generally felt that the environment should be appropriate for the avatar identity (e.g., football field) or activities performed by the avatar (e.g., exercising).

Responses of some participants supported their intention to bring parts of the real world into the virtual environment. Specifically, responses related to viewing the avatar as a friend. For example, one participant stated, "I like a buddy alongside with you as you go, it feels like it changes with you, it sort of a buddy and then is also a teacher [...]."

In alignment with adolescents' responses, we formulated the following design principles:

To allow chronically ill users to achieve extended self-presence, avatars in DHI for self-management of chronic conditions should...

DP8: .... be customizable.

DP9: ... reflect user's vision of future self.

DP10: ... exhibit alignment between avatar identity and virtual environment.

DP11: ...serve as a companion (friend/coach) to a user.

\section{Discussion}

Our study contributes to the knowledge base of avatar design. Existing studies on avatar design either do not focus on design principles at all [50] or focus on design principles that do not capture the concept of self-presence and the context of health [51]. Our study fills in the gap by demonstrating how avatars ought to be designed to achieve self-presence in light of the social context pertinent to user's illness experiences. Specifically, we illustrate how various aspects of user's social context could be captured through avatars to induce self-presence. User's physical functioning, psychological functioning, and illness identity can be reflected in various avatar features (e.g., body features, motivational content provided by avatars, and others). These various aspects of social context align with multiple levels of self-presence (proto, core, extended) and can support personal connection between users and technologies.

Our study has practical implications for the development of DHIs targeting adolescent obesity and 
overweight as well as DHIs targeting other chronic conditions. First, software engineers can use our design principles to guide the development of personally relevant, engaging digital health avatars to be used by obese and overweight adolescents. Specifically, software developers can leverage various layers of selfpresence to design avatars reflective of users. At the body level, avatars can match user's physical characteristics (e.g., weight and height) and physical abilities (e.g., body movements). At the emotion level, avatars can employ a range of emotions to display reactions to healthy vs. unhealthy user behaviors. Finally, at the identity level, avatars can serve as user's friend or model user's future self through various features. In addition, software engineers can use our design principles to evaluate preliminary prototypes of avatars in DHIs for obesity and overweight. Creating DHIs with personally relevant avatars can support user engagement with DHI, thereby leading to health behavior change and improved health outcomes.

Second, software developers and HCI researchers can use our design principles as a reference when designing DHIs for other chronic conditions. Our design principles focus extensively on the social context of users, and, therefore, software engineers and researchers can develop similar principles targeting other health conditions in light of user's social context.

\section{Conclusion}

In this study, a set of theory-driven design principles is derived through the lens of the biocultural theory of presence and the social context of chronically ill users (i.e., physical functioning, psychological functioning, and illness identity). The design principles capture three levels of self-presence, the major concept in the biocultural theory of presence: proto, core, and extended self-presence. At the proto level, avatar design should reflect reflected different components of users' physical functioning (e.g., physical body, body movement). At the core level, avatar design should reflect different components of users' psychological functioning (e.g., motivational content, emotional responses). Finally, at the extended level, avatar design should reflect users' illness identity (e.g., appearance, character, environment).

As user engagement with DHIs remains one of the major issues preventing full realization of the DHI benefits, there is a need to reconsider the design of DHIs and its components. Current DHIs fail to promote the personal relevance of technology and feel relatable to users [33]. thereby leading to problematic user engagement with technology. This study advances the design of DHIs for self-management of chronic conditions by providing a set of design principles for avatars that can contribute to the personal connection between users and technology through self-presence. Improvements in the DHI design have implications for user engagement with technology and lead to improved health outcomes of the chronically ill.

\section{References}

[1] About Chronic Diseases. Secondary About Chronic Diseases, 2019,

https://www.cdc.gov/chronicdisease/about/index.htm.

[2] Murray E, Hekler EB, Andersson G, et al. "Evaluating digital health interventions: key questions and approaches," American Journal of Preventive Medicine, 2016, pp. 843-51

[3] Morton K, Dennison L, May C, et al. "Using digital interventions for self-management of chronic physical health conditions: A meta-ethnography review of published studies." Patient Education and Counseling, pp. 616-35.

[4] Newsom JT, Huguet N, McCarthy MJ, et al. "Health Behavior Change Following Chronic Illness in Middle and Later Life.” Journals of Gerontology: Series B: Psychological Sciences and Social Sciences, 2012, pp. 279-88.

[5] Cole-Lewis H, Ezeanochie N, Turgiss J. "Understanding Health Behavior Technology Engagement: Pathway to Measuring Digital Behavior Change Interventions," JMIR formative research, 2019.

[6] Harris J, Felix L, Miners A, et al. "Adaptive E-Learning to Improve Dietary Behaviour: A Systematic Review and Cost-Effectiveness Analysis," Health technology assessment, 2011, pp.1-160.

[7] Peyrot M, Rubin RR. "Access to Diabetes SelfManagement Education,” The Diabetes educator, 2008, pp. 90-97.

[8] Khadjesari Z, Murray E, Hewitt C, et al. "Can StandAlone Computer-Based Interventions Reduce Alcohol Consumption? A Systematic Review," Addiction, 2011, pp. 267-82.

[9] Pal K, Eastwood S, Michie S, et al. "Computer-based Diabetes Self-Management Interventions for Adults With Type 2 Diabetes Mellitus," The Cochrane database of systematic reviews, 2013.

[10] Andersson E, Ljótsson B, Smit F, et al. "Costeffectiveness of Internet-Based Cognitive Behavior Therapy for Irritable Bowel Syndrome: Results From a Randomized Controlled Trial," BMC public health, 2011.

[11] Kaldo V, Haak T, Buhrman M, et al. "Internet-based Cognitive Behaviour Therapy for Tinnitus Patients Delivered in a Regular Clinical Setting: Outcome and Analysis of Treatment Dropout," Cognitive behaviour therapy, 2013.

[12] Free C, Knight R, Robertson S, et al. "Smoking Cessation Support Delivered via Mobile Phone Text Messaging (txt2stop): A Single-Blind, Randomised Trial," The Lancet, 2011, p.378. 
[13] O’Brien HL, Cairns P, Hall M. “A practical approach to measuring user engagement with the refined user engagement scale (UES) and new UES short form," International Journal of Human - Computer Studies, 2018, pp.28-39.

[14] Csikszentmihalyi, M. Flow: the Psychology of Optimal Performance. New York: University Press, 1990.

[15] Hur I, Cousins KC, Stahl BC. "A critical perspective of engagement in online health communities," European Journal of Information Systems, 2019.

[16] Mahnke R, Benlian A, Hess T. "Flow Experience in Information Systems Research: Revisiting its Conceptualization, Conditions, and Effects," ICIS, 2014.

[17] Rissler R, Nadj M, Adam M. "Flow in Information Systems Research: Review, Integrative Theoretical Framework, and Future Directions," 13th International Conference on Wirtschaftsinformatik, 2017.

[18] Hutton HE, Wilson LM, Apelberg BJ, et al. "A Systematic Review of Randomized Controlled Trials: Web-Based Interventions for Smoking Cessation Among Adolescents, College Students, and Adults." Nicotine \& Tobacco Research, 2011, pp 227-38.

[19] McClure JB, Shortreed SM, Bogart A, et al. "The Effect of Program Design on Engagement With an InternetBased Smoking Intervention: Randomized Factorial Trial," Journal of Medical Internet Research, 2013, pp. 88-100.

[20] Alkhaldi G, Modrow K, Hamilton F, et al. "Promoting Engagement With a Digital Health Intervention (HeLPDiabetes) Using Email and Text Message Prompts: Mixed-Methods Study," Interactive journal of medical research, 2017.

[21] Alkhaldi G, Hamilton FL, Lau R, et al. "The Effectiveness of Prompts to Promote Engagement With Digital Interventions: A Systematic Review," Journal of Medical Internet Research, 2016.

[22] Alice G, Serene Y, Rachel S, et al. "Improving the public health impact of eHealth and mHealth interventions," Australian and New Zealand Journal of Public Health, 2018, pp.118-19.

[23] Blandford A. "HCI for health and wellbeing: Challenges and opportunities," International Journal of Human Computer Studies, 2019, pp. 41-51.

[24] Kelders S, Kok R, Ossebaard H, et al. "Persuasive System Design Does Matter: A Systematic Review of Adherence to Web-Based Interventions," Journal of Medical Internet Research, 2012, pp.17-40.

[25] Christensen H, Griffiths KM, Farrer L. "Adherence in internet interventions for anxiety and depression," Journal of Medical Internet Research, 2009.

[26] Cugelman B, Thelwall M, Dawes P. "Online interventions for social marketing health behavior change campaigns: a meta-analysis of psychological architectures and adherence factors," Journal of Medical Internet Research, 2011.

[27] Neil AL, Batterham P, Christensen H, et al. "Predictors of adherence by adolescents to a cognitive behavior therapy website in school and community-based settings," Journal of Medical Internet Research, 2009.
[28] Perski O, Blandford A, West R, et al. "Conceptualising engagement with digital behaviour change interventions: a systematic review using principles from critical interpretive synthesis," Translational Behavioral Medicine, 2017.

[29] Birnbaum F, Lewis D, Rosen RK, et al. "Patient Engagement and the Design of Digital Health," Academic Emergency Medicine, 2015.

[30] Lupton D. "The digitally engaged patient: Selfmonitoring and self-care in the digital health era," Social Theory \& Health, 2013, pp. 256-70.

[31] Priniski SJ, Hecht CA, Harackiewicz JM. "Making Learning Personally Meaningful: A New Framework for Relevance Research," Journal of Experimental Education, 2018, pp.11-29.

[32] Fogg BJ. Persuasive Technology: Using Computers to Change What We Think and Do. San Francisco, 2003.

[33] Kreps GL. "Achieving the Promise of Digital Health Information Systems," Journal of Public Health Research, 2014.

[34] Schultze U. "Embodiment and presence in virtual worlds: a review," European Journal of Information Systems, 2010, pp. 434-49.

[35] Joo S, Fox J, Hahm JM. "Using Virtual Doppelgängers to Increase Personal Relevance of Health Risk Communication," International Conference on Intelligent Virtual Agents, 2014, pp.1-12.

[36] Fox J, Ahn SJG, Janssen JH, et al. "Avatars Versus Agents: A Meta-Analysis Quantifying the Effect of Agency on Social Influence," Human-Computer Interaction, 2015.

[37] Nowak KL, Fox J. “Avatars and computer-mediated communication: a review of the definitions, uses, and effects of digital representations," Review of Communication Research, 2018.

[38] Biocca F. "The Cyborg's Dilemma: Progressive Embodiment in Virtual Environments," Journal of Computer-Mediated Communication, 1997.

[39] Behm-Morawitz E. "Mirrored selves: The influence of self-presence in a virtual world on health, appearance, and well-being," Computers in Human Behavior, 2013, pp.119-28.

[40] Ratan R, Beyea D, Li BJ, et al. "Avatar characteristics induce users' behavioral conformity with small-tomedium effect sizes: a meta-analysis of the proteus effect," Media Psychology, 2019, pp.651-75.

[41] Hevner AR, March ST, Park J, et al. "Design Science in Information Systems Research," MIS Quarterly, 2004, pp.75-105.

[42] Gregor S, Hevner AR. "Positioning and presenting design science research for maximum impact," MIS Quarterly, 2013, pp.337-355.

[43] Gregor S. "The Nature of Theory in Information Systems," MIS Quarterly, 2006, pp. 611-42.

[44] Gregor S, Jones D. "The Anatomy of a Design Theory," Journal of the Association for Information Systems, 2007, pp. 313-35.

[45] Chandra L, Seidel S, Gregor S. "Prescriptive Knowledge in IS Research: Conceptualizing Design Principles in Terms of Materiality, Action, and 
Boundary Conditions," 48th Hawaii International Conference on System Sciences, 2015, pp. 4039.

[46] Sein MK, Henfridsson O, Purao S, et al. "Action Design Research," MIS Quarterly, 2011, pp.37-56.

[47] Gregor S, Chandra Kruse L, Seidel S. "The anatomy of a design principle," Journal of Association for Information Systems, 2020.

[48] Wieringa RJ. Design Science Methodology for Information Systems and Software Engineering: Springer-Verlag Berlin Heidelberg, 2014.

[49] Karen ME, Elizabeth MB, Caitlin G, et al. "Social Influences, Social Context, and Health Behaviors Among Working-Class, Multi-Ethnic Adults," Health Education \& Behavior, 2007, pp. 315

[50] Rheu MM, Jang Y, Peng W. "Enhancing Healthy Behaviors Through Virtual Self: A Systematic Review of Health Interventions Using Avatars," Games for Health Journal, 2020.

[51] Blake A, Moseley J. "The Emerging Technology of Avatars: Some Educational Considerations. Educational Technology," 2010, pp.13-20.

[52] Riva G, Waterworth JA, Waterworth EL. "The Layers of Presence: A Bio-cultural Approach to Understanding Presence in Natural and Mediated Environments," CyberPsychology \& Behavior, 2004, pp.402-16.

[53] Damasio AR. The feeling of what happens : body and emotion in the making of consciousness. 1st ed. ed: Harcourt Brace, 1999.

[54] Oris L, Luyckx K, Rassart J, et al. "Illness Identity in Adults with a Chronic Illness," Journal of Clinical Psychology in Medical Settings, 2018, pp.429-40.

[55] Ratan R. Self-Presence, Explicated: Body, Emotion, and Identity Extension into the Virtual Self: IGI Global, 2015.

[56] Peeters G, Dobson AJ, Deeg DJH, et al. "A life-course perspective on physical functioning in women," Bulletin of the World Health Organization, 2013, pp. 661-70.

[57] Garber CE, Greaney ML, Riebe D, et al. "Physical and mental health-related correlates of physical function in community dwelling older adults: a cross sectional study," BMC Geriatrics, 2010.

[58] Corbin JM. "The Body in Health and Illness," Qualitative Health Research, 2003.

[59] Turner J, Kelly B. "Emotional dimensions of chronic disease," Western Journal of Medicine, 2000, pp. 124-8.

[60] Psychological Functioning. New York, NY: Springer, 2010.

[61] Oris L, Rassart J, Prikken S, et al. "Illness Identity in Adolescents and Emerging Adults With Type 1

Diabetes: Introducing the Illness Identity Questionnaire," Diabetes Care, 2016, pp. 757-63.

[62] Carroll MD, Navaneelan T, Bryan S, et al. Prevalence of obesity among children and adolescents in the United States and Canada: Hyattsville, MD : U.S. Department of Health and Human Services, Centers for Disease Control and Prevention, National Center for Health Statistics, 2015.

[63] You J, Choo J. "Adolescent Overweight and Obesity: Links to Socioeconomic Status and Fruit and Vegetable
Intakes," International Journal of Environmental Research and Public Health, 2016.

[64] Hruby A, Hu FB. "The epidemiology of obesity: a big picture," PharmacoEconomics, 2015, pp.673

[65] Lee SY. "Examining the factors that influence early adopters' smartphone adoption: The case of college students," Telematics and Informatics, 2014, pp. 30818.

[66] Anderson M, Jiang J. Teens, Social Media \& Technology, 2018. https://www.pewresearch.org/internet/2018/05/31/teenssocial-media-technology-2018/.

[67] Utter J, Larson N, Laska MN, et al. "Self-Perceived Cooking Skills in Emerging Adulthood Predict Better Dietary Behaviors and Intake 10 Years Later: A Longitudinal Study," Journal of Nutrition Education \& Behavior, 2018, pp.494-500.

[68] Lifelong Health. Secondary Lifelong Health. https://developingchild.harvard.edu/science/deepdives/lifelong-health/.

[69] LeRouge C, Dickhut K, Lisetti C, et al. "Engaging adolescents in a computer-based weight management program: avatars and virtual coaches could help," Journal of the American Medical Informatics Association, 2016, pp.19-28.

[70] Knoblock-Hahn AL, LeRouge CM. "A qualitative, exploratory study of predominantly female parental perceptions of consumer health technology use by their overweight and/or obese female adolescent participating in a fee-based 4-week weight-management intervention," Journal of the Academy of Nutrition and Dietetics, 2014, pp. 570-7.

[71] Knoblock-Hahn AL, Wray R, LeRouge CM. "Perceptions of Adolescents with Overweight and Obesity for the Development of User-Centered Design Self-Management Tools within the Context of the Chronic Care Model: A Qualitative Study," Journal of the Academy of Nutrition and Dietetics, 2016, pp. 957-67.

[72] LeRouge C, Durneva P, Sangameswaran S, et al. "Design Guidelines for a Technology-Enabled Nutrition Education Program to Support Overweight and Obese Adolescents: Qualitative User-Centered Design Study," Journal of Medical Internet Research, 2019.

[73] Venkatesh V, Morris MG, Davis GB, et al. "User Acceptance of Information Technology: Toward a Unified View,” MIS Quarterly, 2003, pp. 425-78.

[74] Walls JG, Widmeyer GR, El Sawy OA. "Building an Information System Design Theory for Vigilant EIS," Information Systems Research, 1992, pp. 36-59.

[75] Sorensen G, Emmons K, Hunt MK, et al. "Model for incorporating social context in health behavior interventions: applications for cancer prevention for working-class, multiethnic populations," Preventive Medicine, 2003.

[76] Matavire R, Brown I. "Profiling grounded theory approaches in information systems research," European Journal of Information Systems, 2013, pp. 119-129.

[77] Corbin J, Strauss A. "Grounded Theory Research: Procedures, Canons, and Evaluative Criteria," Qualitative Sociology, 1990. 
[78] Saldaña J. The Coding Manual for Qualitative Researchers. 2nd ed. SAGE; 2013.
[79] Oinas-Kukkonen H. "A foundation for the study of behavior change support systems," Personal and ubiquitous computing, 2018, pp. 1223-1235. 